2015-05

Passing crisis and emergency risk communications: The effects of communication channel, information type, and repetition

Edworthy, Judy

http://hdl.handle.net/10026.1/3269

10.1016/j.apergo.2014.12.009

Applied Ergonomics

Elsevier BV

All content in PEARL is protected by copyright law. Author manuscripts are made available in accordance with publisher policies. Please cite only the published version using the details provided on the item record or document. In the absence of an open licence (e.g. Creative Commons), permissions for further reuse of content should be sought from the publisher or author. 


\title{
Passing crisis and emergency risk communications: The effects of communication channel, information type, and repetition
}

\author{
Judy Edworthy ${ }^{a}$, ${ }^{*}$ Elizabeth Hellier ${ }^{a}$, Lex Newbold ${ }^{b}$, Kirsteen Titchener ${ }^{c}$ \\ ${ }^{a}$ Cognition Institute, University of Plymouth, Drake Circus, Plymouth, Devon, UK \\ ${ }^{\mathrm{b}}$ University of Exeter, Exeter, Devon, UK \\ ${ }^{c}$ Plymouth, Devon, UK
}

\section{A R T I C L E I N F O}

\section{Article history:}

Received 9 July 2014

Accepted 16 December 2014

Available online

\section{Keywords:}

Communication channel

Warning design

Crisis and emergency risk communications

\begin{abstract}
A B S T R A C T
Three experiments explore several factors which influence information transmission when warning messages are passed from person to person. In Experiment 1, messages were passed down chains of participants using five different modes of communication. Written communication channels resulted in more accurate message transmission than verbal. In addition, some elements of the message endured further down the chain than others. Experiment 2 largely replicated these effects and also demonstrated that simple repetition of a message eliminated differences between written and spoken communication. In a final field experiment, chains of participants passed information however they wanted to, with the proviso that half of the chains could not use telephones. Here, the lack of ability to use a telephone did not affect accuracy, but did slow down the speed of transmission from the recipient of the message to the last person in the chain. Implications of the findings for crisis and emergency risk communication are discussed.
\end{abstract}

(C) 2015 Elsevier Ltd and The Ergonomics Society. All rights reserved.
Behavior in response to civil emergencies is a topic of considerable concern. Across the world people are vulnerable to risks ranging from naturally occurring events such as earthquakes, extreme weather conditions, and tsunamis, to industrial and other accidents, and of course terrorist attacks. Responding to such emergencies is a topic of significant concern and investment from governments, who are increasingly involving psychologists, human factors and other experts, and knowledge of human behavior in their preparedness plans. There are many important areas of research that are relevant, such as people's understanding of risks, for example their willingness and ability to respond to an emergency; their trust in different sources of information; and the way the information spreads through a community. Much of the direct and indirect research that is relevant here has been distilled into broad-ranging guidance and reviews for policy makers and other stakeholders in emergency preparedness and is embodied in the emergent field of Crisis and Emergency Risk Communication (CERC, e.g. Sorensen, 2000; Reynolds and Seeger 2005;Seeger, 2006; Reynolds, 2002; Wood et al. 2008; Centers for Disease Control and Prevention (CDCP), 2012).

\footnotetext{
* Corresponding author.

E-mail address: jedworthy@plymouth.ac.uk (J. Edworthy).
}

The Crisis and Emergency Risk Communication model is intended as a tool to assist in the management of civil emergencies across the whole range of activities. Reynolds and Seeger (2005) present the working model of CERC as consisting of five main stages: pre-crisis; initial event; maintenance; resolution; and evaluation. All of those aspects of human behavior listed above, and many others, are relevant at various points in the model. In this study we are concerned with behavior which is relevant to specific aspects of CERC at the precrisis and initial event stages - the development of messages and the understanding of channels and methods of communication (Reynolds and Seeger, 2005).

One important aspect of the initial stages of an emergency is that emergency messages will be sent out from central sources (probably multiple sources, in a variety of formats) and will be passed on from person to person. In the three studies reported here we simulate this process. Specifically, we investigate the relationship between the mode of message transmission (the communication channel), the accuracy of transmission of the elements of messages down a chain of receivers, the effect of repeating and/or enhancing the messages and (in the final study), the speed and accuracy of transmission of messages in a more realistic setting where some channels might be unavailable. 
Research which has investigated the transmission of information from one person to another demonstrates, not surprisingly, that information degrades as it is passed from person to person. Information that might be initially quite detailed tends to be reduced quite quickly to a few key facts (Allport and Postman, 1947). Though there is initial degradation, beyond four or five people messages tend not to degrade further (Dalrymple, 1978). Research has little to say about the relative survival of different elements of crisis messages under controlled conditions when passed from person to person, but is informed by research both on warning message design and studies which have looked post-hoc at real emergencies.

Because message information will inevitably be lost during transmission, it is important that they are designed in such a way as to be maximally efficient, especially if they are transmitted via a medium which restricts the size of the message (such as Twitter). In essence the accepted guidance is that a message should have everything in it that the receiver needs, and nothing that he or she does not, stated clearly and unambiguously. The CDC (2012) has proposed 'Who, What, Where, When, Why and How' as representing the essential components of a crisis communication. Similarly, Mileti and Sorensen (1990) propose that specific pieces of information in warning messages should include the hazard, the location, the time of the incident, and guidance. Reynolds and Seeger (2005) suggest that there are clear distinctions between more general risk communications and crisis communications, but warnings are more broadly recommended to have four main components: a signal word (e.g. 'Danger'), a statement of the hazard, a statement of the consequences of exposure and instructions on how to avoid the hazard. These are designated in ANSI standard Z.535 (1987). We therefore based the messages we tested on the collective guidance above.

Though the research literature has little to say about the relative survival of the individual elements of messages as it passes down chains of people under controlled conditions, studies which have looked post-hoc at the way information is disseminated under real crises can inform this topic (Sutton et al., 2014; Butts et al., 2007; Chew and Eysenbach, 2010). For example, Sutton et al. (2014) looked at the pattern of tweeting and retweeting messages during a 48-h period during a canyon fire in 2012. They also carried out a content analysis of the tweets and found that the messages most likely to be retweeted were not only more likely to be hazardrelated, but were more likely to concern hazard impact and to be advisory in nature. Thus this data suggests that key elements 'survive' in terms of their likelihood of being passed on through retweeting. Whether or not those elements survive when they are passed from person to person is one of the main focuses of the studies presented here.

As well the transmission of information from one person to another and the relative survival of message elements down those chains, we are interested in how both of those factors might be affected by the mode of transmission of the message. In the first two studies we ask participants to pass messages down chains by means of speaking, writing, email, SMS text messaging or telephone. The most obvious dichotomy here is that some of these modes are primarily auditory, while the others are primarily visual. Studies comparing visual and auditory materials in applied settings have demonstrated mixed effects. Conway and Christiansen (2005) showed that auditory stimuli displayed a learning advantage over visual stimuli, and Wogalter and Young (1991) found that auditory warning messages were more readily complied with than visual ones. This study also showed that presenting a warning in both visual and auditory modalities outperformed the individual modalities, as did Cao et al. (2010). In terms of direct memory effects, Furnham et al. (1990) found that printed factual information was better remembered than an audio-visual or audio presentation, whereas Corston and Colman (1997) found that audio and print forms of communicating warnings increased recall relative to video format.

Obviously there are clear stimulus differences between written and spoken communications which might underpin any observed differences between them, such as the relative permanence of a written stimulus, which can be re-examined at will, compared with the typically fleeting nature of a spoken message. However, with modern communication methods the distinction between auditorily-presented information (such as speech) and visually presented information (such as an SMS message) is rather more blurred. Biber (1991) points out that speech and writing are not bimodal, but instead vary along a number of dimensions such as interactive vs edited, and reported vs immediate. These dimensions appear to vary across different styles of communication which ostensibly use the same mode. For example, as Herring points out (2010) many authors have suggested that computermediated communication, especially messaging, is more like conversation than written communication, though it has the property of permanence. This is likely to be true also of SMS messaging. Also, people are more likely to exhibit certain types of behavior in some modes than in others - for example, Whitty et al. (2012) demonstrated that people are more likely to lie spontaneously on the telephone, but to tell planned lies via SMS. Thus we do not necessarily expect all versions of a single mode (primarily auditory or primarily visual) to demonstrate the same characteristics. For example, messages may not be transmitted the same way when written down on paper than when passed on via SMS. In the studies that follow we attempt to control for the most obvious and potentially confounding factors across the modes tested, but our key aim is to provide a fair test of the differences when the modes function in the way they typically would do in reality.

In the first experiment, five communication channels are used. These are SMS text, email, paper, face-to-face, and telephone. They are compared by passing a set of messages down a chain of respondents under laboratory conditions. In the second laboratory study, messages are presented either by telephone or are written, with and without repetition, with some participants being given instructions either to re-read and check what they have written or to ask questions in order to reduce the a priori advantages that one or other communication might possess over the other. In the final field experiment, participants received one of two types of auditory message and were asked to pass it on. In some cases they were allowed to use whatever means they preferred to communicate the message down the chain, and in others they were restricted from using the telephone, as they might be in a real civil emergency.

\section{Experiment 1}

The aim of this experiment was to explore both the effect of communication channel on the transmission of information through chains of participants and the relative persistence of the various message elements down the chain. Five communication channels were compared - two primarily auditory (face-to-face and cellphone) and three primarily written (SMS text message, email, and paper). These channels were selected to best represent the communication options available in emergency incidents. We used chains of ten people as this represents a long chain (for example, Dalrymple (1978) found that natural message chains seemed to consist of eight or fewer people). If there is to be breakdown of information, we would expect this to have happened before the information has reached the end of the chain. 


\section{Method}

\subsection{Experimental design}

Five different warning messages were constructed using the same format and structure, but containing information about different hazards (see Materials). Each of the messages was prepared in the five modes to be tested so that the messages were identical other than the mode of presentation. Five groups of ten participants were recruited. Within each group of ten participants, each modality was used once and each message was used once, so that each message/modality combination was unique for each group. The combining of the messages with modalities was different for each of the five groups. Across the five groups, each message was combined with each of the modalities so that across the experiment, each message was presented in each modality once, in a total of 25 presentations of the messages across the five groups.

\subsection{Participants}

There were 50 participants ( 14 males, 36 females) aged 18-53 years ( $M=22.5$ years). They were recruited through the University of Plymouth participant pool and were paid $£ 10$ for participation.

\subsection{Materials}

Five messages were constructed from 7 structural elements. These were; a Signal word, Substance, Source, Location, Symptom 1, Symptom 2 and Advice. For example, 'Warning! There has been a release of Gas 32 into large water pipes in the Peterborough area. This is likely to cause nausea and infant convulsions. We advise you to drink bottled water.' Four other messages were created, each using the same structure but containing different details for each element. In addition, four cellphones, two tape recorders with detachable microphones and two computers connected to the Internet were also required.

\subsection{Procedure}

In each session, a group of 10 participants was tested. There were five groups of 10 participants in total across sessions. In each session the group of 10 participants passed all five messages between members in a designated participant order, using each of the five communication channels once. The designated order of participants in the information chain was varied between each message/channel. Each participant in the chain was required to try to remember enough information to pass the message on to the next participant without referring back to the original source. Thus in the written conditions they were able to read the message as often as they needed to and in the spoken conditions they were able to ask questions of the transmitter. The experimenter observed all proceedings.

In the paper condition, the first participant entered the lab where the warning was printed on a piece of A4 paper on their desk. They were asked to read the message and then hand the paper with the message back to the experimenter. Their task was to restate the message by handwriting it on the paper provided. This paper was then left on the desk for the next participant. The procedure was repeated down the chain of 10 participants, with each successive participant handing the written message they had received to the experimenter before writing their own version of the message for the next participant. If the writing was considered illegible by the experimenter, the writer was asked to rewrite the message. This occurred only once or twice during the study.
In the email condition, email accounts were set up for the participants. The first participant logged on to their account to read a message that had already been sent to them. They were asked to restate the message in an email and send it to the next participant. Participants were not able to refer back to the message once they had started writing their own version of it. The procedure was repeated down the chain of 10 participants.

In the SMS condition, the message was sent to the first participant's cellphone. They were instructed to read the message, to create a text message restating the information and send it to the next participant in a different room. This procedure was repeated down the chain of participants. Participants were able to use predictive text messaging if they wished, and to use text-speak.

In the face-to-face condition the experimenter and first participant entered the laboratory and the experimenter passed the message to the participant verbally. The experimenter then left the room and was replaced by the second participant. The first participant passed the message to the second and then left the room, to be replaced by participant 3 and so on, down the chain of 10 participants.

In the phone condition, the experimenter phoned the first participant with the warning message. After the experimenter had hung up the participant was required to phone the next participant (in a different room) and pass on the message. The first participant then returned to the waiting room to be replaced by participant 3 . This procedure was repeated down the chain of 10 participants. Both the face-to-face and phone conditions were recorded.

\subsection{Results}

\subsubsection{Data analysis}

The seven message elements were coded for transmission accuracy by two independent coders. Each message element was coded 2 (the element was there in its original form), 1 (the element was there but had been altered) or 0 (a completely missing element). Thus, higher scores indicated more accurate messages or elements thereof. Words added to the messages were also counted. Inter-rater reliability was calculated using Cohen's Kappa, Kappa $=0.89$ which is above the 0.7 cut off for acceptable agreement between raters.

This data was then subject to further analysis as presented in the following sections.

\subsection{Transmission accuracy by communication channel and element}

A 2-way channel $\times$ element ANOVA revealed a significant main effect for Channel $(F[4,42]=54.77, p<0.001)$. Paper messages were most accurately transmitted, $(M=1.13)$ followed by SMS $(M=1.01)$, email $(M=0.91)$, face-to-face $(M=0.69)$ and phone $(M$ $=0.65$ ). Significant differences (Bonferroni) were found between paper and all other channels (all at $p<0.001$ except between paper and SMS, $p<0.05$ ) and between SMS and both phone and face-toface $(p<0.001)$.

A significant main effect was also found for message element ( $F$ $[4.49,40]=144.42, p<0.001)$. 'Substance' and 'Advice' were the most accurately transmitted elements $(M=1.25)$ followed by 'Location' $(M=1.21)$, 'Symptom 1' $(M=0.89)$, 'Symptom 2' $(M=0.71)$, 'Signal word' $(M=0.62)$ and 'Source' $(M=0.23)$. Bonferroni tests showed significant differences between 'Substance' and 'Source', 'Signal word', and 'Symptom 1 and 2' $(p<0.01)$. Both 'Advice' and 'Location' were transmitted more accurately than 'Source', 'Signal word', 'Symptom 1' and 'Symptom 2 ' $(p<0.01)$. 'Symptom 1' was transmitted more accurately than 'Source', 'Signal word' and 'Symptom 2' ( $p<0.01)$,'Symptom 2' was transmitted more accurately than 'Signal word' and 'Source' 


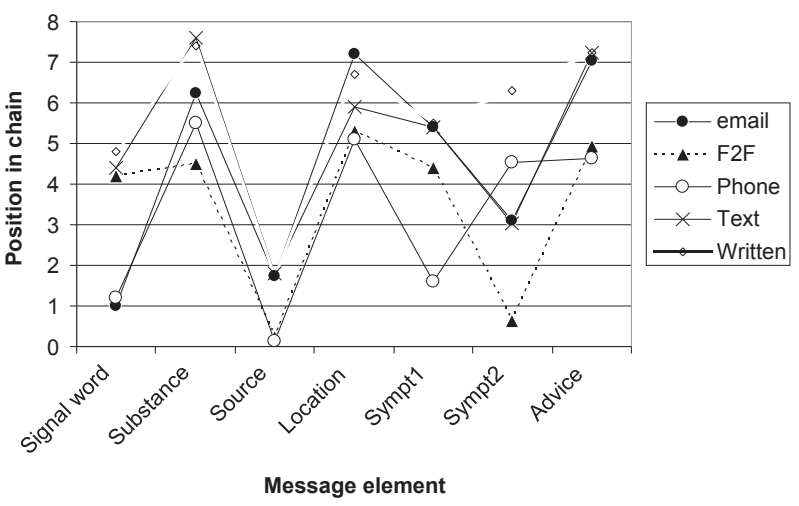

Fig. 1. Average survival value of elements by communication channel (Experiment 1).

$(p<0.01)$ and 'Signal word' was transmitted more accurately than 'Source' $(p<0.01)$.

\subsection{Element survival}

The mean scores for element accuracy can be approximated into a score, which demonstrates how long a word typically survives down the chain of participants. The assumption made is that once an element has disappeared in a communication it does not return. Each mean element score is out of 2, so multiplying this score by 10 (number of people in the chain) and dividing by 2 gives the approximate point in the chain where the element is lost. Fig. 1 plots the mean survival of each message element down the chain of participants, as a function of communication channel. Overall, the elements, 'Substance', 'Location' and 'Advice' survive furthest down the chain, whereas 'Source' is typically lost within one or two people. In addition, the survival of some elements depends on communication channel; for example, 'Signal word' typically survives to the fifth person in the chain in the paper condition but only the first person in the chain in the phone condition.

\subsection{Communication down the chain}

The overall accuracy with which each message was communicated down the chain was calculated by collapsing across participants and elements. Table 1 demonstrates that the mean scores fall as position in the chain increased, showing that information is lost at each stage. A two-way communication channel $\times$ position ANOVA showed a main effect for channel $(F[4,37]=27.3, p<0.01)$, a main effect for position $(F[9,37]=17.14, p<0.01)$ but no significant interaction between the two $(F[36,160]=1.38, p=0.094)$. Performance generally fell as position in the chain increased, and performance was better using some channels than others.

Table 1

Mean accuracy at each position in the chain for each communication channel (Experiment 1).

\begin{tabular}{lllllll}
\hline Position in chain & Email & F2F & Phone & SMS & Paper & Mean \\
\hline 1 & 49.4 & 39.6 & 41.6 & 41.0 & 53.6 & 45.04 \\
2 & 41.6 & 28.4 & 31.4 & 37.8 & 49.4 & 37.72 \\
3 & 32.0 & 26.2 & 28.4 & 32.8 & 45.5 & 32.98 \\
4 & 29.2 & 24.6 & 25.6 & 31.2 & 37.4 & 29.60 \\
5 & 29.2 & 19.0 & 25.4 & 30.4 & 32.2 & 27.24 \\
6 & 26.8 & 20.6 & 21.6 & 29.6 & 29.2 & 25.56 \\
7 & 27.2 & 20.2 & 20.4 & 25.2 & 27.8 & 24.16 \\
8 & 24.4 & 20.0 & 18.8 & 22.8 & 26.0 & 22.40 \\
9 & 22.0 & 18.0 & 17.0 & 22.2 & 24.4 & 20.72 \\
10 & 30.6 & 23.6 & 14.6 & 21.2 & 22.0 & 22.40 \\
Mean & 31.26 & 24.02 & 24.48 & 29.42 & 34.75 & \\
\hline
\end{tabular}

A series of ANOVAs were carried out for each stage in the chain. These demonstrated a significant effect of communication channel through positions 1 to 4 in the chain. At position 1, there was a significant effect of communication channel $(F[4]=5.1)$ with significant differences between paper and face-to-face $(F[1]=28.4$, $p<0.01)$, paper and phone $(F[1]=12.31, p<0.01)$ and paper and SMS $(F[1]=12.4, p<0.01)$. At position 2 , there was again a significant effect of communication channel $(F[4]=5.25, p<0.01)$, with significant differences between paper and face-to-face $(F$ $[1]=50.1, p<0.01)$, paper and phone $(F[1]=21.5, p<0.01)$ and paper and $\operatorname{SMS}(F[1]=7.4, p=0.053)$. At position 3 there was still a significant effect of channel $(F[4]=3.56, p<0.01)$ with significant differences between paper and face-to-face $(F[1]=68, p<0.01)$ and paper and phone $(F[1]=16.7, p<0.01)$. At position 4 , there was no effect of communication channel $(F[4]=1.83, p=0.172$, neither was there at position $5(F[4]=2.11, p<0.01)$, position $6(F$ $[4]=2.05, p<0.01$ ) or beyond. At the first link in the chain, all communication channels other than email are less accurate than the paper communication, however by the 4 th position, differences between channels are no longer significant. This indicates that the degradations in message accuracy occur in the first few transmissions, and that after the fourth or fifth person in the chain the accuracy of transmission stabilizes as the information is lost.

\subsection{Additional words}

An additional words measure was also used, which quantifies an alternative source of inaccuracy, information added to the message. A repeated measures ANOVA $(F[4,42]=19.28, p<0.001)$ revealed a significant effect of communication channel on the number of additional words used. The mean number of additional words added across the entire chain, per participant, were 7.15 for paper, 5.25 for email, 5.75 for SMS, 9.93 for face-to-face and 14.7 for phone. Bonferroni post-hoc tests showed that these differences again reflect differences between spoken and written methods of delivery. Listed in descending size of mean difference, there was a significant difference between Phone and Email, Phone and SMS, Phone and Paper, Face-to-face and Email, and between Face-to-face and SMS (all at $p<0.01$ ). The number of words added to a message appears to correspond negatively with the accuracy with which the original message is preserved. To some extent, participants appear to add words in order to preserve the approximate length of the original message.

\subsection{Discussion}

The results show three main findings: that different communication channels vary in their reliability in terms of how accurately people transmit a message down a chain; that different elements of a message are more or less resistant to being omitted; and that the early advantages shown by some channels are lost further down the chain. The advantage of written channels over spoken ones is demonstrated both when accuracy is measured by information lost from messages and also when measured by new information added. So participants both lose information, and embellish more, in spoken communications.

For the written communication channels there was scope for participants to re-read the information before they restated the message. As our studies seek to provide a 'fair test' of practical differences between the modes, participants in the spoken conditions were allowed to ask questions, as a way of embellishing the information in a way that one might if the written mode was unavailable. In the written conditions they could re-read the message. In each case, the participants' operational task was to derive enough information from the message to pass it on. We found 
however that participants were reluctant to ask questions in the spoken conditions whereas re-reading the document did not require them to interact with anyone, so this might have given some advantage to the written form of the message. In the second experiment we control for exposure, and allow participants to enhance the information gained through methods appropriate to the visual and auditory modality.

The other key finding in this study is that elements of the warning message differ in how accurately they are transmitted. Typically 'Location', 'Advice', and 'Substance' survived well regardless of communication channel. The key elements to be lost are the source of the release, the listed symptoms, and the signal word. In practical terms it is the most important, behaviordetermining, aspects of warning messages that have survived; the location of the risk, the advice, and the substance that has been released. Whether these elements survive because of the structure of the message or because of receivers' mental model of which information is important, is not clear. Recall the ANSI Z.535 recommendations and earlier observation that four key components of warning messages can provide a framework for understanding warning messages. The substance, its source and its location can be construed as the hazard; symptoms can be construed as consequences; and advice can be construed as instructions. Experiment 1 demonstrates that, as in the study by Wogalter et al (1987), signal words can be forgotten because they are subsumed in the message itself. Whilst the substance and its location are readily remembered and passed on, source is readily lost; thus some elements of the hazard are passed on while others are lost. In this study the 'Source' generally referred to the precise location of the problem such as 'rivers' or 'large water pipes'. This would seem to be vital information, so it is interesting that while people could remember and pass on what the hazard was and where it was geographically, they tended not to remember its precise location. The results also showed that 'Symptoms' tended to be less well passed on. In terms of the four main components of a warning message these can be construed as consequences, which, as Wogalter et al. (1987) showed, are either less important or are subsumed in the rest of the message. Finally, 'Advice' is remembered as well as 'Location' and 'Substance' and can be construed as instructions.

The final key finding of this study was that, inevitably, message accuracy degrades fastest at the beginning of an information chain, and later losses in accuracy are smaller. At the same time, messages are embellished in those conditions where the information lost is greatest, though in many cases the embellishment still carried the sense of the message. Our findings also indicate that though we did not expressly find an interaction between communication channel and position in the chain, the advantage for written versions of the message are lost at about the fourth position. Thus if a receiver is receiving a fourth- or greater-hand message, the modality in which it is conveyed is no longer relevant.

\section{Experiment 2}

In the first experiment there was a clear advantage for the written mode when the messages were presented in a single exposure. In the second experiment we compare written (paper) and spoken (face-to-face) communications when they are either repeated, or participants are encouraged to enhance their understanding of the message, or both.

A considerable amount of research has been carried out on the relationship between repeated exposure and dependent variables which might affect either aesthetic responses or future behavior. Much of this has focused on the relationship between repeated exposure, complexity, and aesthetic responses (following Berlyne's two-factor theory $(1970,1971)$. Many studies have used the two- factor theory as a way of looking at the persuasiveness of communications (e.g. Cacioppo and Petty, 1979), and some have looked specifically at its role in advertising (e.g. Childers, 1986; Anand and Sternthal, 1990). Whilst our studies are not concerned with persuasiveness, complexity is of some importance as it is possible that our messages, because they have several components, may require several exposures to be fully remembered. Underpinning the inverted-U relationship between complexity, repetition, and liking (Berlyne, 1971), is the sheer ability of participants to process the elements of the stimuli used. Repeated exposure makes it more possible for participants to process those elements. It is quite possible therefore that differences between the written and the spoken presentations of the message in Experiment 1 can be accounted for partly because the message was too complex for participants to take in fully in one exposure. Additionally, in the first study, the participants would in principle have been able to read the written message more than once. Our observations also suggest that participants when receiving a message were reluctant to ask questions of the transmitter of the message. Thus in the second study we also exert greater control on the availability of the stimuli to the participant.

As Biber (1991) points out, the differences between written and spoken communication are not as clear-cut as we might think. In our experiments however the distinction is quite clear, as we use written and spoken communications in the more typical bimodal sense. Our written messages were presented to participants on a sheet of paper, and our spoken messages were conveyed to the participants in a face-to-face situation, without the use of any writing materials. In Experiment 2 we present both written and spoken messages either once or twice i.e. with or without repetition. We added a second condition intended to further enhance the participants' understanding of the message but in a way commensurate with the modality in question, as we wished to provide a fair test of any differences between the two modes. In some conditions, participants were asked explicitly to check their understanding of the message either by re-reading what they had written (written messages) or by questioning the speaker (spoken messages) as much as required. We should anticipate little advantage of either communication channel once repetition and clarification opportunity is controlled for.

The first experiment also showed that some elements of a message are better relayed than others. Here we explore whether those effects are replicated and whether they are influenced by the experimental variables of repetition and instructions to clarify the message.

\subsection{Method}

\subsubsection{Experimental design}

This was a mixed design. The two between-subjects factors were repetition of the message (repetition/no repetition) and enhancement (re-reading in the case of the written condition and questioning the speaker in the spoken condition). Mode (written, spoken) was a within-subjects factor. There were 20 groups of up to five participants, each of whom passed two messages down the chain, one in written form and one in spoken form. Each of the two messages tested (see below) was presented to half of the groups in a spoken form and half in written form. Additionally, half of the chains passed down the spoken message first and half the written message.

\subsubsection{Participants}

173 participants took part, 50 males and 123 females, aged between 18 and 49 years, ( $M=21.4$ years). Some participants were psychology undergraduates who took part for course credit and 
some were recruited through advertisements placed across the university campus and paid $£ 5$.

\subsubsection{Materials}

Two warning messages were used, each with 7 elements (Substance, Source, Location, Symptom 1, Symptom 2, Advice $1 \&$ Advice 2 ). Some minor changes were made to the messages in comparison with those previously used. The signal word was removed and a second piece of advice inserted instead. 'Substance' was made more generic e.g. 'weak acid' rather than e.g. 'Factor NX'as feedback from Experiment 1 suggested that unfamiliar names might distract participants from other message content. The two messages were as follows:

- There has been a release of chemical fertilizer into rivers near farmland in the Exeter area. It is likely to cause skin irritation and nausea. It is advised that you keep children indoors and seek medical advice.

- There has been a release of weak acid into small water pipes in the Cornwall area. It is likely to cause vomiting and muscle cramps. It is advised that you inform elderly relatives and avoid tap water.

In addition, a tape recorder with detachable microphone, blank paper and pens were required.

\subsubsection{Procedure}

Participants were randomly assigned to a position in a chain of up to 5 participants. Each chain took part in one between-subject condition (no enhancement/no repetition; no enhancement/repetition; enhancement/no repetition or enhancement/repetition). Within each between-subject condition, participants passed on both warning messages, and used both spoken and written communication channels. The order of the written and spoken conditions and the message/mode pairing was counterbalanced across the groups (see Experimental Design, above). The order of the participants within a chain was changed between the first and the second message. The procedure for each communication channel is described below. An experimenter observed the sessions.

\subsubsection{Spoken}

The first participant sat in the laboratory opposite the experimenter. The experimenter read the message out loud (with or without repetition and enhancement, depending on condition). This experimenter then left the room and was replaced by the second participant. The first participant passed the message (with or without repetition and enhancement, depending on condition) to participant two by speaking it. This procedure was repeated down the chain of participants. The last participant passed the message back to the experimenter who had earlier delivered the first message. The sessions were recorded. In the enhancement conditions participants were told, 'Feel free to ask questions and clarify the message if necessary. Make sure you have understood the message and think you can remember it before attempting to pass it on'. The participant was told to ensure they conveyed the instructions to seek clarification to the next participant.

\subsubsection{Written}

Participants sat down in front of the message, which was typed on A4 paper. The instructions asked them to read the message (with repetition and enhancement depending on condition) and then reproduce it on the paper provided. Once completed, the participant left the room. The next participant entered and was asked to read, and then reproduce, the message that the previous participant had left. This procedure was repeated down the chain of participants. In the enhancement conditions participants were told, 'Before leaving the message make sure that you are happy with it, re-read it, check its accuracy and that it includes all the information'.

\subsection{Results}

\subsubsection{Data analysis}

The seven message elements were coded for transmission accuracy by two independent coders. Each message element was coded 2 (the element was there in its original form), 1 (the element was there but had been altered) or 0 (a completely missing element). Thus, higher scores indicated more accurate messages or elements thereof. Words added to the messages were also counted. Inter-rater reliability was calculated using Cohen's Kappa, Kappa $=0.85$ which is above the 0.7 cut off for acceptable agreement between raters.

This data was then subject to further analysis as presented in the following sections.

\subsubsection{Transmission accuracy scores}

Table 2 shows accuracy scores as a function of communication channel, repetition, and enhancement, collapsed across elements.

A mixed ANOVA, 2 (repetition) $\times 2$ (enhancement) $\times 2$ (communication channel) $\times 7$ (elements), identified a main effect for communication channel, $(F[1,162]=44.72, p<0.001)$, where the written delivery was more accurate than the spoken delivery. There was also an effect of repetition $(F[1,162]=24.19, p<0.001)$, whereby messages that were repeated were transmitted more accurately. There was an interaction between communication channel and repetition $(F[1,162]=4.70, p=0.03)$. Post hoc analysis (Bonferroni) indicated a significant difference only $(p<0.01)$ between the spoken/no repetition and all other conditions. Thus, repetition had a more beneficial effect on accuracy for spoken messages than for written ones. There was no main effect of enhancement, but there was a main effect of message element $(F[6$, $157]=43.26, p<0.001$ ). The means for each of the elements were; 1.35 ('Substance'), 0.69 ('Source'), 1.26 ('Location'), 0.96 ('Symptom 1'), 1.02 ('Symptom 2'), 0.97 ('Advice 1') and 0.67 ('Advice 2'). Post hoc (Bonferroni) comparisons indicated that means were significantly higher for 'Substance' than for all elements other than 'Location' ( $p<0.01)$; significantly higher for 'Location' than all elements other than 'Substance' $(p<0.01)$; significantly higher for 'Symptom 2' than 'Advice 2' and 'Source' $(p<0.01)$; and significantly higher for 'Symptom 1' and 'Advice 1' than 'Advice 2' and 'Source' $(p<0.01)$.

There were some interactions involving the elements. There was a two-way interaction between communication channel and elements $(F[6,157]=23.3, p<0.01)$. Means for each of the seven elements by communication channel were 1.18 (face-to-face) and 1.52 (paper) for 'Substance'; 0.53 and 0.86 for 'Source'; 1.27 and 1.25 for 'Location'; 0.90 and 1.02 for 'Symptom 1'; 0.51 and 1.52 for

Table 2

Mean transmission accuracy scores by communication channel, repetition and instructions (Experiment 2).

\begin{tabular}{llll}
\hline & No repetition & Repetition & Mean \\
\hline Face-to-face, no instruction & 0.73 & 0.90 & 0.82 \\
Face-to-face, instruction & 0.67 & 1.09 & 0.88 \\
Written, no instruction & 1.14 & 1.19 & 1.17 \\
Written, instruction & 0.99 & 1.18 & 1.09 \\
Mean & 0.88 & 1.09 & \\
\hline
\end{tabular}


'Symptom 2'; 0.96 and 0.99 for 'Advice 1 ' and 0.60 and 0.74 for 'Advice 2'. Thus the scores for the paper-based communications were generally higher than for spoken, but much higher for 'Symptom 2'. There were three-way interactions between elements, repetition and enhancement $(F[6,157]=4.78, p<0.01)$, and element, communication channel and repetition $(F[6,157]=2.7$, $p<0.01$ ). Table 3 shows the results of the element $\times$ communication channel $\times$ repetition interaction. The table shows that retention of some elements was far more consistent across channel and repetition than others. For example, the scores for 'Substance' were fairly consistent across the four conditions whereas 'Symptom 2 ' showed a large increase in mean score between the no repetition and the repetition conditions, which is demonstrated less obviously for most of the other conditions. There was a four-way communication channel $\times$ element $\times$ repetition $\times$ enhancement interaction $(F[6157]=2.21, p<0.05)$.

\subsection{Added words}

A measure of added words was taken, as in Experiment 1. The mean number of added words, averaged across participants, was 13.26 for the spoken messages and 8.19 for the written messages $(t$ $[165]=7.05, p<0.001)$.

\subsection{Discussion}

This experiment has demonstrated again an advantage for communicating warning messages through written channels. However, this advantage is dramatically reduced when the message is repeated, as there appears to be a greater advantage to repeating a spoken message than there does to reading a written message more than once.

The added benefit of hearing a message a second time, plus the facility to interact with the speaker of the message, appears to improve performance with spoken communications to the level of written ones. The various interactions found in Experiment 2 can be largely attributed to the improvement in performance when the receiver is allowed to hear the spoken version of the message more than once, and the further improvement (though not statistically significant) when they are additionally allowed to quiz the speaker.

Experiment 2 also largely replicated the findings for message elements found in Experiment 1. The message elements 'Substance' and 'Location' were most accurately transmitted, and 'Source' and 'Advice 2' the least. 'Location' has survived well across both experiments and 'Source' has fared badly. The fact that 'Substance' and 'Location' appear to be well transmitted might be explained as a primacy effect, but as 'Source', which appears in a similar serial position, appears to be easily lost it is more likely that the effect is attributable to the receiver retaining the important information at the expense of the less important (suggesting a cognitive rather

\section{Table 3}

Mean scores for the element $\mathrm{x}$ communication channel $\times$ repetition interaction (Experiment 2).

\begin{tabular}{|c|c|c|c|c|c|}
\hline & Face-to-face & Paper & Face-to-face & Paper & Mean \\
\hline & No repetition & No repetition & Repetition & Repetition & \\
\hline Substance & 1.02 & 1.54 & 1.33 & 1.49 & 1.35 \\
\hline Source & 0.49 & 0.81 & 0.56 & 0.90 & 0.69 \\
\hline Location & 1.09 & 1.12 & 1.45 & 1.38 & 1.26 \\
\hline Symptom 1 & 0.64 & 0.85 & 1.15 & 1.19 & 0.96 \\
\hline Symptom 2 & 0.33 & 1.63 & 0.69 & 1.41 & 1.02 \\
\hline Advice 1 & 0.86 & 0.81 & 1.06 & 1.16 & 0.97 \\
\hline Advice 2 & 0.49 & 0.73 & 0.72 & 0.75 & 0.67 \\
\hline Mean & 0.70 & 1.07 & 0.99 & 1.18 & \\
\hline
\end{tabular}

than a perceptual effect). Loss of 'Advice 2' in this study is a concern as advice is one of the most important features of a message and can be construed as part of the instructions. The effects of adding further advice elements, and ways of improving the survivability of advice elements, might be considered in future research.

The evidence from Experiments 1 and 2 suggests that there are some features of warning messages which are better remembered and conveyed than others. There is also something of a pattern whereby those elements most poorly conveyed with a single exposure to the message are conveyed more readily, but still relatively poorly, when the message is repeated. This is generally more evident in the spoken conditions than in the written conditions.

Because our studies were laboratory-based, it was not possible to get a measure of the speed with which the messages were conveyed, which is clearly important for real communications. In the final experiment we also consider how fast a message spreads between groups of acquaintances in the real world, as a function of communication channel. We were interested in simulating a reallife scenario and so we also constrained the way in which some of the participants could communicate. In a real civil emergency people are sometimes either unable to, or advised not to, use telephones. Thus in the final experiment we introduce a condition where participants are not allowed to use their telephone.

\section{Experiment 3}

In the third experiment we use a more realistic 'real world' paradigm in addressing similar questions to Experiments 1 and 2. In order to simulate some important real world conditions we sometimes restricted the use of the telephone - a situation which might mirror a real-life situation in which either the telephones are out of action, or the public have been asked not to use them - and we also measure the speed with which a message passes down the chain in addition to measuring its accuracy. For ethical reasons however we could not use emergency messages as we had in the first two studies, for while the participants themselves would have known the messages to be part of the study, we could not risk those messages spreading beyond the participant group. In the final study, we presented only an auditory message to the first participant in the chain. This was either in the form of a telephone message or as a pre-recorded CD. Both types of presentation simulate an important method of communicating actual emergencies to the public; telephoning one another is what people would often do during an emergency (if allowed), and a CD can be loosely thought of as simulating a radio or TV broadcast.

In this study we recruited groups of individuals known to one another (either a family or a set of friends) who, once briefed, were set to receive a set of messages at random times and were then asked to convey the message down their own chain. We presented the initial message either via the telephone or via a $\mathrm{CD}$ sent to their address, awaiting activation when instructed. Other than controlling the way in which the message was conveyed to the first recipient, we did not control how participants communicated with one another other than restricting half of the participants to any medium other than the telephone (for both speaking and SMS messaging). The messages we used had a similar structure (i.e. possessed comparable individual elements) to those used in Experiments 1 and 2 but contained innocuous content.

\subsection{Method}

\subsubsection{Experimental design}

The experiment was a $2 \times 2$ mixed design. The within subjects factor was method of message delivery to the group (CD vs. phone) and the between subjects factor was method of communication 
(free or restricted). The dependent variables were accuracy of the message and the time taken to pass the message from the experimenter around the group to the last participant.

\subsubsection{Participants}

Sixty-four people took participated in the experiment. They were aged between 18 and 61 years with a mean age of 24.6 years. Seventeen were male and 47 were female. All participants were recruited by flyers posted on campus at the University of Plymouth or through the University of Plymouth Participant Pool, and each was paid $£ 20$. The participants were recruited in groups of four people (16 groups). Each group was required to consist of friends or family, living within a $10 \mathrm{~km}$ radius of each other, who had a working cellphone, access to a means of playing a $C D$ and who consented to have their contact details released to other members of their group (email address, address, landline and cellphone numbers etc). The requirement that group members lived geographically close to one another was to ensure that members could communicate with each other face-to-face if they wished to do so.

\subsubsection{Materials}

Since participants in this study were recruited partly from the general public and the messages were sent at unpredictable times, messages with innocuous content were used. The structure of the messages was designed to mirror the elements of those used previously, so that each element performed the same function as the messages tested in Experiments 1 and 2.

Eight messages were created with the same structure. The specific corollaries to the messages used previously and their general corollary to the four components of warning statements are as follows: The name of the person the message was about (source/ hazard); the group they came with (source/hazard); the event they were at (substance/hazard); the venue where the event was being held (location/hazard); what they brought with them (symptom/ consequences); what they did while they were there (symptom/ consequences); what advice the organizers had for next time (advice/instructions); and the reason for the advice (advice/instructions). For example, 'Graham Cook and his neighbors attended a senior ballroom dance held at the Thomas Theatre. Some people brought digital cameras and many drank too much. The organizers advised that for future events people should come by coach to avoid the delays travelling home'.

Each participant was given a $\mathrm{CD}$ (containing only the message that the participant would have to send on at some later point), a sheet of contact details for members of their group and instructions for completing the experiment. An Envox phone recording system was used to record the messages that participants received from other participants.

\subsubsection{Procedure}

Each group received all eight messages, half by phone and half by $C D$ (in the $C D$ condition the initial message recipient was phoned and told to listen to their $C D$ as soon as possible after the call), counterbalanced across groups. The order in which groups received the messages and method of message delivery was also counterbalanced. In the $\mathrm{CD}$ condition the initial receiver could listen to the message as many times as they wanted to, and in the phone condition they were allowed to ask questions and interact with the caller.

The initial message recipient in a group was chosen at random for each message (pre-selected before the CDs went out), and asked to ensure that the message was passed around as fast as possible. Participants had to phone the Envox system every time they received a message, stating the message they had received, when they received it, how it was delivered and by whom. Half of the groups were allowed to pass the message among themselves in any way they liked (free), the other half were not allowed to use the telephone (restricted). Once each member of the group had received a message (or $72 \mathrm{~h}$ had elapsed, the timeout period) the group was notified that the trial for that message was complete. The next message was sent to the group during the following day. Before the message trials began, a test message was sent to every participant.

\subsection{Results}

\subsubsection{Data analysis}

The primary dependent measures of interest were transmission accuracy, transmission time and the relative decay of different message elements. The messages recorded on the Envox system were coded for accuracy. Each message element scored as either 1 (correct) or 0 (incorrect or missing). An element was scored as correct if the meaning of the information was retained even if different words were used. Transmission speed was measured as the time taken to pass a message from the experimenter to the last of the four participants in a group. Timing began when the experimenter passed the message to the initial receiver and finished when the last receiver phoned the Envox system. Groups failing to complete the transmission of a message within $72 \mathrm{~h}$ were timed out and $72 \mathrm{~h}$ was entered as their time. We did not enter the data into a complete 3-way analysis, because individual cell size for method of delivery $\mathrm{x}$ method of communication $\times$ element was relatively small, and because there was no control over the communication channels used. We present first the effects of method of delivery and method of communication, collapsed across element. The second part of the analysis considers the effects of elements.

\subsection{Message transmission accuracy}

Mean message accuracy scores (maximum 1), as a function of delivery and communication methods were 0.789 (CD/free), 0.616 (phone/free), 0.677 (CD/restricted) and 0.657 (phone/restricted). A $2 \times 2$ mixed ANOVA revealed a significant main effect for method of delivery $(F[1,12]=8.163, p=<0.05)$, so that messages delivered initially by $C D$ were transmitted more accurately than those delivered by phone. There was no effect of free vs. restricted on accuracy $(F[1,12]=0.190, p=0.671)$ and no interactions.

The position of the message in the sequence of messages (form 1 to 8 , as each chain conveyed a total of eight messages) for each group was also considered. There was no effect of position ( $F[7$, $42]=1.385, p=.237$ ) on transmission accuracy, so participants neither improved nor got worse at the task as the experiment progressed. The effect of message approached significance, ( $F[7$, $42]=2.225, p=<0.051)$, with messages 2 and 5 being transmitted more accurately $(M=0.813$ and $M=0.792$ respectively) and message 8 transmitted least accurately $(M=.576)$. No interactions were found.

\subsection{Message transmission speed}

Message transmission times (in minutes), as a function of delivery and communication method were; 697 (CD/free), 697 (phone/free), 3017 (CD/restricted) and 2113 (phone/restricted). A $2 \times 2$ mixed ANOVA found no effect of delivery method on speed ( $F$ $[2,12]=1.029, p=0.330)$. There was an effect of method of communication $(F[1,12]=22.821, p=<0.01)$, so that messages transmitted freely were transmitted more quickly than those transmitted without using the phone. No interaction was found. 


\subsection{Transmission accuracy of different message elements}

The mean accuracy scores for elements, collapsed across messages and conditions, were 0.87 (Name), 0.84 (Group), 0.74 (Event), 0.56 (Venue), 0.63 (Brought), 0.70 (Did), 0.87 (Advice) and 0.67 (Reason). A one-way within-subjects ANOVA confirmed a significant effect of element on accuracy $(F[6.21,2976)]=48.902$, $p<0.01$ ). Post hoc pair-wise comparisons (Bonferroni) revealed significant differences in the accuracy with which elements were transmitted between all pairs of elements $(p<0.01)$ except between: 'Name' and 'Group'; 'Name' and 'Advice'; 'Group' and 'Advice'; 'Event' and 'Did'; 'Venue' and 'Brought'; 'Venue' and 'Reason'; 'Brought' and 'Did'; 'Brought' and 'Reason'; and 'Did ' and 'Reason'.

\subsection{Choice of communication channel}

In this study, participants chose how to communicate within their group (notwithstanding the restricted condition, where they could not select the telephone either for speaking or for SMS messaging), and the method used was recorded for most of the 384 instances of a message passing from one participant to another. Table 4 shows that when people can choose freely, they use SMStext messaging or the telephone to transmit messages. When they are not allowed to use the telephone they choose primarily email communication, followed by face-to-face communication.

\subsection{Discussion}

Experiment 3 demonstrates that the way in which a message is delivered to the first person in a chain influences the accuracy with which the message is conveyed, but not the speed. The speed of transmission down the chain is influenced by whether or not a telephone is used.

Restricting participants to methods of communication other than the telephone appears to have helped sustain the accuracy of the message. Table 4 indicates that whilst face-to-face communication (a less accurate method) was used more often in the restricted versus the free condition, there was a much larger favoring of email and social networking sites (more accurate) in the restricted condition in comparison with the free condition. We do not know whether participants simply forwarded emails to one another when they used email; if they did, however, this would be adaptive behavior and suggests again that participants maximize the accuracy of the message by whatever means available. Table 4 also shows that in the free condition participants favored SMS messaging over the telephone - again suggesting a desire to convey the message as accurately as possible by using a written rather than spoken medium. Whilst we do not know the extent to which taking such decisions was something of which the participants were consciously aware, our data does suggest adaptive behavior of this type.

This experiment also shows that communications restricted to methods other than the telephone are conveyed more slowly. There was enormous variability in the time taken for a message to move down the chain, resulting in large mean times for message

\section{Table 4}

Frequency with which different communication channels were selected (Experiment 3).

\begin{tabular}{lllllc}
\hline & Email & F2F & Phone & SMS & Social network sites \\
\hline Free & 28 & 36 & 45 & 72 & 2 \\
Restricted & 74 & 54 & - & - & 29 \\
\hline
\end{tabular}

transmission. Much of this is probably due to the innocuous nature of the messages coupled with the fact that participants did not know when a message would be transmitted and therefore might be engaged with other tasks such as work, or might be away from their cellphone and/or computer.

Finally, Experiment 3 also demonstrated that different elements of the message varied in how well they were remembered. In terms of specific comparisons with Experiments 1 and 2, what was construed to be 'Source' information was much better conveyed. Participants remembered 'Name' and 'Group' better than they remembered 'Event' (the corollary of 'Substance' and 'Venue' (the corollary of 'Location'). They did however remember 'Advice' (the corollary of 'Advice') better, though the 'Reason' tended to be lost. They conveyed 'Brought' and 'Did' less well - the corollaries of 'Symptoms'- which were also not well conveyed in Experiments 1 and 2. However, we cannot make too much of this as the corollaries are not obvious and the messages used here had no personal relevance to the participants. It would be reasonable to assume that if participants really had been attending the events they would have made more effort to remember what they were. In terms of the broader categories of hazard, consequence and information statements then the results show the same general pattern in that hazard and information statements tend to be remembered (and therefore conveyed) better than consequence statements.

\subsection{General discussion}

The studies reported here demonstrate that when messages are passed down chains of people, some elements of the messages survive better than others; that some modes are more accurate than others, but some are faster; that there are early advantages in the use of some modes, which dies out by position four in the chain; and that repetition and enhancement of messages improves the accuracy of transmission, especially for spoken messages.

That some elements survive better than others suggests either that the better-remembered elements are simply more memorable than others, or that participants made more effort to remember some elements of the message because they assigned a greater importance to them. As we used a number of messages which might be more or less memorable than one another (and have more or less memorable elements), the latter is more likely. Like Wogalter et al. (1987), we found that hazard and instruction information is generally better passed on than signal words and consequences. The reason we propose is similar to that cited by Wogalter et al. (1987), which is that signal words and consequences are subsumed and can be inferred from the hazard and instruction statements (and thus do not need restating). Similar findings and conclusions have been found in other studies using different approaches. Naire and Panderirada (2011) report the phenomenon of survival processing - whereby participants preferentially remember information important for their survival: ultimately this may be what is happening in our studies. We can also infer that participants were conveying information according to its utility. Where it was difficult for them to remember all of the elements, they passed on the information that could not be inferred from other information. This is demonstrated most clearly in the spoken conditions; where the message was repeated there was significant improvement in the accuracy with which the poorly performing information was passed on. This suggests that memory capacity was restricting participants from remembering everything that they wanted to remember and pass on.

In their study of Twitter posting and re-posting after the Waldo Canyon wildfire, Sutton et al. (2014) demonstrated that the content of the most re-tweeted messages were concerned with guidance and impact themes, particularly advisory and location information. 
Obviously the restriction to 140 characters means that only one or two pieces of information can be imparted in a single tweet, so the various central themes were spread across the tweets rather than being embodied in single tweets. Overall, Sutton et al. demonstrated that message content, message style and message exposure all had effects on re-tweet rates. Thus there are now a range of methodologies and approaches which indicate that people, when under pressure, can successfully convey the elements of emergency messages most central to their survival. Neither our study nor the Sutton et al. study demonstrates whether or not the messages are ultimately effective, and result in better safety behavior than would occur without those messages. We also do not know whether the loss of information down the chains of participants tested in our studies, or the fragmented messages sent via twitter has any effect on safety behavior. Behavioral measures are very difficult to take, for several reasons. In the real world one does not know the baseline level of safety behavior which would occur without such messages (and modern methods of communication such as twitter means that we cannot control people communicating, so could never establish a baseline anyway) and in the laboratory, where it is possible to measure behavioral endpoints (such as in Edworthy et al., 2004) the risks have to be fabricated, for ethical reasons, which may affect the outcome. Subjective measures of compliance also do not necessarily reflect the degree to which actual compliance may occur. However, this is an important topic for future research.

These experiments demonstrate a number of findings with implications for the broadcast and transmission of crisis and emergency risk communications. The CERC literature (e.g. CDCP, 2012) commonly recommends that emergency responders choose the appropriate communication channel, and here have added issues of message accuracy and element survival to the criteria against which a communication channel might be assessed. Our findings suggest that written communications generally facilitate more accurate transmission of information than spoken communications, as we would expect. This is true when accuracy is measured as information lost from a message as it passes down a chain and also as information added to a message. Our final experiment suggests that communicating via telephone may be faster, but at the expense of accuracy. However, advances in technology make the distinction between written and spoken communication ever more blurred, making possible communication methods which take advantage of both the speed of verbal communication and the reliability and permanence of written communication. Our experiments also show that when a spoken message is repeated and enhanced, the advantage of written communication largely disappears.

In any case, differences in performance between communication channels were only present in the earlier parts of the chain by the fifth person the differences had disappeared. Research in other areas, using less constrained paradigms, suggests also that the number of links in naturally occurring message chains is relatively small. For example, Dalrymple's study (1978) of the way rumor spread after a crisis demonstrated that rumors chains typically consisted of fewer than eight people. The 'small world' paradigm (Travers and Milgram, 1969; White and Houseman, 2003), shows that when people are required to communicate with a named (but unknown to them) individual, this can usually be done in six or fewer links. Our experiments suggest that large chains of communicators are unwieldy and unreliable - luckily, they appear not to be typical of what happens in real life.

Experiments 1 and 2 dictated which mode was allowable when participants passed the message down the chain. However, Experiment 3 showed that when participants could choose how to communicate with one another, they chose to use the telephone or
SMS text messaging. Our findings also suggest that restricting the use of the telephone, as might be necessary or desirable in a civil emergency, will result in more accurate (but slower) message transmission. One interesting observation is that when people are able to use phones, they have a preference for SMS messaging and when they cannot use the telephone, they have a preference for emailing. Thus in both cases they are optimizing the accuracy of their messages by using written, permanent methods of communication.

Finally, it is important to reiterate that our findings are concerned with message accuracy (and to some extent speed) and are not concerned with compliance (or subjective compliance), understanding, persuasion, comprehension or any other aspect of behavior directly connected with emergency messages. Neither are they concerned with factors which might affect the efficacy of the initial message, such as trust in the source and/or believability of the message. These other areas are of course of great importance and are the topics of many other studies. Many of these issues are also embodied within the CERC guidance and are thus recognized as being important. The work reported here is concerned with the beginning of all of those processes, as none of them can begin without the receiver being able to pass on a message accurately.

\section{Acknowledgments}

This research was wholly supported by a UK Home Office grant 'Factors influencing information spread and reliability', awarded to Elizabeth Hellier \& Judy Edworthy.

\section{References}

Allport, G.W., Postman, L., 1947. The Psychology of Rumor. Holt, Rinehart \& Winston, New York.

Anand, P., Sternthal, B., 1990. Ease of message processing as a moderator of repetition effects in advertising. J. Market. Res. XXVII, 345-353.

ANSI Z535 (1987), 1987. Criteria for Safety Symbols. American National Standards Institute.

Berlyne, D.E., 1970. Novelty, complexity, and hedonic value. Percept. Psychophys. 8 (5), 279-286.

Berlyne, D.E., 1971. Aesthetics and Psychobiology. Appleton-Century-Crofts, East Norwalk, CT, US.

Biber, D., 1991. Variation across Speech and Writing. Cambridge University Press.

Butts, C.T., Petrescu-Prahova, M., Cross, B., 2007. Responder communication networks in the World Trade Center Disaster: implications for modelling of communication within emergency settings. Math. Sociol. 31 (2), 121-147.

Cacioppo, J.T., Petty, R.E., 1979. Effects of message repetition and position on cognitive response, recall and persuasion. J. Personal. Soc. Psychol. 37, 97-109.

Centers for Disease Control and Prevention (CDCP), 2012. Crisis and emergency risk communication. http://emergency.cdc.gov/cerc/pdf/CERC_2012edition.pdf (accessed May 2014).

Chew, C., Eysenbach, G., 2010. Pandemics in the age of Twitter: content analysis of tweets during the 2009 H1N1 outbreak. PLoS One 5, 11. http://dx.doi.org/ 10.1371/journal.pone.0014118.

Cao, Y., Mahr, A., Castronovo, S., Theune, M., Stahl, C., Muller, C., 2010. Local danger warnings for drivers: the effect of modality and level of assistance on driver reaction. In: Proceedings of the 15th International Conference on Intelligent User Interfaces, pp. 239-248.

Conway, C.M., Christiansen, M.H., 2005. Modality-constrained statistical learning of tactile, visual and auditory sequences. J. Exp. Psychol.: Learn. Mem. Cognit. 31 (1), 24-39.

Childers, T.L., 1986. Assessment of the psychometric properties of an opinion leadership scale. J. Market. Res. XXIII, 184-188.

Corston, R., Colman, A.M., 1997. Modality of communication and recall of healthrelated information. J. Health Psychol. 2 (2), 185-194.

Dalrymple, C.F., 1978. The flow of crisis information as a probe of work relations. Cah. Can. Sociol. 3, 71-88.

Edworthy, J., Hellier, E., Morley, N., Grey, C., Aldrich, K., Lee, A., 2004. Linguistic and location effects in compliance with pesticide warning labels for amateur and professional users. Hum. Factors 46 (1), 11-31.

Furnham, A., Gunter, B., Green, A., 1990. Remembering science: the recall of factual information as a function of the presentation mode. Appl. Cogn. Psychol. 4, 203-212.

Herring, S.C., 2010. Computer-mediated conversation: introduction and overview Language@Internet 7, article 2 urn:nbn:de:0009-7-28011. 
Mileti, D.S., Sorensen, J.H., 1990. Communication of Emergency Public Warnings. Oak Ridge National Laboratory, Colorado State University.

Naire, J., Panderirada, J., 2011. Congruity effects in the survival processing paradigm. J. Exp. Psychol. Learn. Mem. Cognit. 37 (2), 539-549.

Reynolds, B., 2002. Crisis and Emergency Risk Communication. Centers for Disease Control and Prevention, Atlanta, GA.

Reynolds, B., Seeger, M., 2005. Crisis and emergency risk communication as an integrative model. J. Health Commun. 10, 43-55.

Seeger, M., 2006. Best practice in crisis communication - an expert panel process. J. Appl. Commun. Res. 34 (3), 232-244.

Sorensen, May 2000. Hazard warning systems. Nat. Hazards Rev. 119-125.

Sutton, J., Spiro, E.S., Johnson, B., Fitzhugh, S., Gibson, B., Butts, C.T., 2014. Warning tweets: serial transissiom of messages during the warning phase of a disaster event. Inform. Commun. Soc. 17 (6), 765-787.

Travers, J., Milgram, S., 1969. An experimental study of the small world problem. Sociometry $32(4), 425-443$.
White, D.R., Houseman, M., 2003. The navigability of strong ties: small worlds, tie strength and network topology. Complexity 8 (1), 72-81.

Whitty, M.T., Buchanan, T., Joinson, A.N., Meredith, A., 2012. Not all lies are spontaneous: an examination of deception across different modes of communication. J. Am. Soc. Inform. Sci. Technol. 63 (1), 208-216.

Wogalter, M.S., Young, S., 1991. Behavioral compliancexe "compliance" to voice and print warnings. Ergonomics 34, 78-89.

Wogalter, M., Godfrey, S., Fontenelle, G., Desaulniers, D., Rothstein, P., Laughery, K. 1987. Effectiveness of warnings. Hum. Fact. 29 (5), 599-612.

Wood, M., Kano, M., Mileti, D., Bourque, L.B., 2008. Questionnaire specifications: Documentation of the national survey of disaster experiences and preparedness (NSDEP). Report of the National Consortium for the Study of Terrorism and Responses to Terrorism (START) Working Group III. Southern California Injury Prevention Research Centre, Los Angeles. http://www.sscnet.ucla.edu/issr/da/ earthquake/NCSTART/NCSTARTSurvSpecsFINALwAppdxCSecure-3.pdf (accessed May 2014) 